\title{
Spontaneous spinal epidural abscess in patients 50 years of age and older: a 15-year institutional perspective and review of the literature
}

\author{
Clinical article
}

\author{
Owoicho Adogwa, M.D., M.P.H., ${ }^{1}$ IsaAC O. Karikari, M.D., ${ }^{1}$ Kevin R. CARr, M.D., ${ }^{2}$ \\ Max Krucoff, M.D., ${ }^{1}$ Divya Ajay, M.D. ${ }^{1}$ Parastou Fatemi, B.S., ${ }^{1}$ Edgar L. Perez, B.S., ${ }^{1}$ \\ Joseph S. Cheng, M.D., M.S., ${ }^{3}$ Carlos A. Bagley, M.D., ${ }^{1}$ And Robert E. Isaacs, M.D. ${ }^{1}$ \\ ${ }^{1}$ Division of Neurosurgery, Department of Surgery, Duke University Medical Center, Durham, North \\ Carolina; ${ }^{2}$ Department of Neurological Surgery, University of Texas Health Sciences Center at San Antonio, \\ Texas; and ${ }^{3}$ Department of Neurological Surgery, Vanderbilt University Medical Center, Nashville, Tennessee
}

\begin{abstract}
Object. A spinal epidural abscess (SEA) is a rare but severe infection requiring prompt recognition and management. The incidence of SEA has doubled in the past decade, owing to an aging population and to increased use of spinal instrumentation and vascular access. The optimal management of SEAs in patients 50 years of age and older remains a matter of considerable debate. In an older patient population with multiple comorbidities, whether intravenous antibiotics alone or in combination with surgery lead to superior outcomes remains unknown. The present study retrospectively analyzes cases of SEAs, in patients 50 years of age and older, treated at Duke University Medical Center over the past 15 years.

Methods. Eighty-two patients underwent treatment for a spinal epidural abscess between 1999 and 2013. There were 46 men and 36 women, whose overall mean age ( \pm SD) was $65 \pm 8.58$ years (range $50-82$ years). The mean duration of clinical follow-up was $41.38 \pm 86.48$ weeks. Thirty patients $(37 \%)$ underwent surgery for removal of the abscess, whereas $52(63 \%)$ were treated more conservatively, undergoing CT-guided aspiration or receiving antibiotics alone based on the results of blood cultures. The correlation between pretreatment variables and outcomes was evaluated in a multivariate regression analysis.

Results. Back pain and severe motor deficits were the most common presenting symptoms. Compared with baseline neurological status, the majority of patients (68\%) reported being neurologically "better" or "unchanged." Twelve patients (15\%) had a good outcome (7 [23\%] treated operatively vs $5[10 \%]$ treated nonoperatively, $\mathrm{p}=0.03)$, while clinical status in 41 patients $(50 \%)$ remained unchanged (10 [33\%] treated operatively vs 31 [60\%] treated nonoperatively, $\mathrm{p}=0.01)$. Overall, 20 patients $(25 \%)$ died $(9[30 \%]$ treated operatively vs 11 [21\%] treated nonoperatively, $p=0.43$ ). In a multivariate logistic regression model, an increasing baseline level of pain, the presence of paraplegia or quadriplegia on initial presentation, and a dorsally located SEA were independently associated with poor outcomes.

Conclusions. The results of the study suggest that in patients 50 years of age and older, early surgical decompression combined with intravenous antimicrobial therapy was not associated with superior clinical outcomes when compared with intravenous antimicrobial therapy alone.
\end{abstract} (http://thejns.org/doi/abs/10.3171/2013.11 .SPINE13527)

\section{Key Words • ventral • dorsal • conservative management • intravenous antibiotics $\quad$ spinal epidural abscess $\bullet \quad$ infection}

$\mathrm{S}$ PINAL epidural abscesses (SEAs) are rare but serious infections located between the spinal dura mater and the vertebral periosteum. They often require emergency neurosurgical intervention to avoid permanent neurological deficits. Over the past several decades this condition has drawn significant attention due to its

Abbreviation used in this paper: SEA = spinal epidural abscess. increasing incidence (doubling from 0.2 to 2 cases per 10,000 hospital admissions from 1947 to 1974 to $2-3$ cases per 10,000 hospital admissions) $)^{1,8,19,21}$ and continued high mortality rate, ranging from $15 \%$ to $23 \% .^{10,16}$

Treatment options for SEAs traditionally consist of laminectomy to allow surgical drainage and decompression, CT-guided percutaneous aspiration, prolonged antibiotic therapy, or a combination of these approaches. Whether prolonged antibiotic therapy alone or in com- 


\section{Management of spinal epidural abscesses in older patients}

bination with surgery leads to superior clinical outcomes remains unknown. Several authors have attempted to stratify patients into treatment groups by pretreatment neurological status, 7,17,20 anatomy of the SEA (dorsal vs ventral) ${ }^{11}$ patient age, and other clinical characteristics, ${ }^{4}$ with varying results.

Spinal epidural abscesses have been reported to occur in patients who range in age from 10 days to 87 years, with most cases occurring in those $30-60$ years old. ${ }^{16}$ Not surprisingly, increasing patient age has been shown to be a poor prognostic factor. ${ }^{4}$ However, to date, no published studies have investigated treatment options in patients age 50 years and older presenting with an SEA. Due to the unique needs of older patients, as well as the multitude of variables that affect outcomes related to age, we feel it is necessary to examine data from an older population in isolation to help guide clinical decision making for these patients. Therefore, this study retrospectively analyzed cases of SEA, in patients 50 years of age and older, treated at our institution over the past 15 years.

\section{Methods}

\section{Patient Selection}

From January 1, 1999, to April 31, 2013, 82 patients who were at least 50 years of age were treated at Duke University Medical Center for a spontaneous epidural abscess. The institutional review board approved this study. Patients were identified using a computerized search for the ICD-9-CM code 324.1 (abscess of any part of the spinal cord). The inclusion criteria were the identification of pathological tissue (phlegmon, pus, or both) in the epidural space by surgery or biopsy. Patients with a known history of postoperative infection were excluded.

Patient demographics, clinical presentation, comorbidities, radiological studies, abscess location, and all treatment variables were reviewed for each case.

\section{Diagnostic Evaluation}

A spontaneous epidural abscess was principally suspected on clinical grounds based on the clinical history, physical examination findings, and/or laboratory findings and was confirmed with radiological studies. All patients enrolled in this study had standard laboratory tests on admission to the hospital including the following: erythrocyte sedimentation rate, peripheral white blood cell counts, C-reactive protein, complete urine analysis, microbiology, and blood cultures. The erythrocyte sedimentation rate was determined by the Westergren method and was considered abnormal if it was greater than 15 $\mathrm{mm}$ /hour; C-reactive protein was considered abnormal if it was greater than $5 \mathrm{mg} / \mathrm{dl}$. Bacterial identification and susceptibility testing were performed according to the guidelines of the Centers for Disease Control.

On presentation to the emergency department, the majority of patients underwent contrast-enhanced CT scanning. Because a CT scan does not always provide conclusive evidence of SEA, the diagnosis was definitively established in all patients by MRI with and without contrast.

\section{Duration of Symptoms and Extent of Preoperative Neurological Deficit}

Patients with symptom manifestations lasting less than 2 weeks prior to hospital admission were considered to have an acute presentation. Those whose symptoms lasted greater than 2 weeks prior to presentation were considered to have a more chronic course. Patients were stratified based on the extent of their neurological deficit on hospital admission; patients were categorized as having no deficits (Group 1), mild radiculopathy (Group 2), or paraplegia or quadriplegia (Group 3).

\section{Performance of Surgical Decompression Versus Antibiotics} Based on Surgeon "Preference"

The decision to treat a confirmed SEA with antibiotics or surgical decompression or both was at the discretion of the attending neurosurgeon, after taking into consideration all pertinent comorbidities, patient age, and the extent of the epidural abscess. Nevertheless, all surgeons involved in our study practiced similar postintervention management paradigms, with daily clinical examination, laboratory workup, and antibiotic administration.

\section{Statistical Analysis}

Parametric data are expressed as the mean \pm SD and were compared utilizing the Student t-test. Nonparametric data are expressed as the median (interquartile range) and were compared utilizing the Mann-Whitney U-test. Nominal data were compared with the chi-square test. Univariate analysis (JMP 6, SAS Institute) was performed to identify independent predictors of poor treatment outcomes. Variables associated with poor treatment outcomes $(\mathrm{p}<0.05)$ in univariate analyses were then included in a multivariate logistic regression model. Variables in the multivariate analysis with $\mathrm{p}<0.05$ were considered significant (JMP 6, SAS Institute).

\section{Results}

From January 1, 1999, to April 31, 2013, 82 patients age 50 years and older were treated at Duke University Medical Center for a spontaneous epidural abscess. There were 46 men and 36 women. The overall mean age ( \pm SD) was $65 \pm 8.58$ years (Group 1, $65 \pm 8$ years; Group 2, 64 \pm 8 years; and Group 3, $64 \pm 10$ years; $p=0.43$ ) (Table 1). Forty-six patients (56\%) were Caucasian and 33 (40\%) were African American (Table 1). On contrast-enhanced MRI of the spine, the most common levels affected were L-3 (22\% of patients), L-4 (33\% of patients), and L-5 (31\% of patients). The median number of vertebral levels involved was 2 (IQR 3-5). The majority of imaged SEAs extended continuously across vertebral levels, with the exception of 1 patient with both a 5-level cervicothoracic SEA and a 2-level lumbar SEA.

\section{Clinical Features and Risk Factors for the Development of an SEA}

Table 2 summarizes the most common predisposing factors for the development of an SEA: 25 patients (30\%) had multiple medical problems, 31 patients (38\%) had a 
O. Adogwa et al.

TABLE 1: Baseline demographics and characteristics of patients with spontaneous SEAs*

\begin{tabular}{lc}
\hline Characteristics & Value \\
\hline mean age (yrs) & $65 \pm 8.58$ \\
male (\%) & $46(56)$ \\
race (\%) & \\
African American & $33(40)$ \\
Caucasian & $46(56)$ \\
Hispanic & $1(1)$ \\
Asian & $1(1)$ \\
other & $1(1)$ \\
anatomical location (\%) & \\
cervical & $10(12)$ \\
thoracic & $31(38)$ \\
lumbar & $41(50)$ \\
mean follow-up (wks) & $41.38 \pm 86.48$ \\
\hline
\end{tabular}

* Mean values are presented \pm SD.

history of diabetes mellitus, 20 (24\%) had end-stage renal disease, 7 (9\%) had endocarditis, $5(6 \%)$ had a recent history of urosepsis, 4 (5\%) had rheumatoid arthritis and were on immunosuppressive therapy, 2 (2.4\%) had a history of spinal trauma, and 1 patient was HIV positive.

\section{Symptoms and Findings on Presentation}

The patients' presenting complaint(s) ranged in severity from nonspecific back pain to marked weakness. As previously stated, patients were stratified into 3 groups based on the degree of neurological deficit: Group 1 (no neurological deficit), Group 2 (mild radiculopathy), and Group 3 (paraplegia or quadriplegia). There were 47 (57\%) patients in Group 1, 23 (28\%) in Group 2, and 12 $(15 \%)$ in Group 3. Back pain and severe motor deficits were the most common presenting symptoms, occurring in $76 \%$ and $57 \%$ of patients, respectively (Table 3); fever was present in 52\%; urinary/bowel dysfunction was present in $22 \%$; and encephalopathy was present in $6 \%$.

TABLE 2: Predisposing risk factors for the development of spontaneous SEAs

\begin{tabular}{lc}
\hline \multicolumn{1}{c}{ Risk Factor } & No. of Patients $(\%)$ \\
\hline diabetes mellitus & $31(38)$ \\
end-stage renal disease & $20(24)$ \\
endocarditis & $7(9)$ \\
urosepsis & $5(6)$ \\
intravenous illicit drug use & $1(1.2)$ \\
rheumatoid arthritis & $4(5)$ \\
previous spinal procedure & $2(2.4)$ \\
previous spinal trauma & $3(3.6)$ \\
previous dental trauma & $2(2.4)$ \\
alcoholic or viral cirrhosis & $4(4.8)$ \\
HIV & $1(1.2)$ \\
\hline
\end{tabular}

TABLE 3: Symptoms and signs at diagnosis of SEAs

\begin{tabular}{lc}
\hline \multicolumn{1}{c}{ Finding } & No. of Patients (\%) \\
\hline back pain & $62(76)$ \\
motor weakness & $47(57)$ \\
sensory abnormalities & $35(43)$ \\
fever & $43(52)$ \\
urinary/bowel dysfunction & $18(22)$ \\
encephalopathy & $5(6)$ \\
\hline
\end{tabular}

\section{Radiographic Findings}

Magnetic resonance imaging with/without contrast adminstration revealed an SEA in all 82 patients. The imaging characteristics of an epidural abscess are quite distinctive. An epidural mass appears iso- or hypointense on T1-weighted MRI compared with the spinal cord and is hyperintense on proton-density and T2-weighted imaging sequences. Coexisting low signal changes in adjacent vertebral bodies are often seen on T1-weighted MRI with high signal intensity in the intervertebral disc and vertebral bodies on T1-weighted MRI sequences.

\section{Treatment}

All patients received a prolonged course of intravenous antibiotics, which were initiated on an empirical basis and later tailored to the particular organism. Treatment options included CT-guided aspiration or blood cultures followed by prolonged intravenous antibiotics or surgical decompression with intravenous antibiotics. Generally, surgery was recommended for patients presenting with a neurological deficit (Grade 2 or 3) except in instances in which paraplegia or quadriplegia was present for longer than 48 hours. Additionally, surgery was recommended if there was evidence of a progressive spinal deformity even in the absence of a neurological deficit. Of the 82 patients, $30(37 \%)$ underwent surgery for removal of the abscess, whereas $52(63 \%)$ were treated more conservatively with CT-guided aspiration or examination of blood cultures to establish the pathogen involved. Of those requiring surgery, 16 patients (53\%) underwent simple laminectomy, 7 (23\%) underwent laminectomy with fusion, 3 (10\%) underwent a transpedicular decompression, and 4 (13\%) underwent anterior decompression (with or without corpectomy) and fusion.

\section{Pathogen Causing Spontaneous Epidural Abscess}

The causative pathogens were determined from intraoperative sampling, CT-guided aspiration, or blood cultures in 72 patients $(88 \%)$ (Table 4). The most common pathogens causing SEA were methicillin-sensitive Staphylococcus aureus (28\%), methicillin-resistant S. aureus (28\%), coagulase-negative Staphylococcus species (11\%), and Enterococcus (4\%). Other causative organisms included Streptococcus species (5\%) and Abiotrophia (1\%). The causative organism was unknown in 10 patients (12\%).

\section{Clinical Follow-Up}

The mean duration of clinical follow-up was $41.38 \pm$ 
Management of spinal epidural abscesses in older patients

TABLE 4: Underlying proven pathogen causing SEA in 82 patients

\begin{tabular}{lc}
\hline \multicolumn{1}{c}{ Pathogen } & No. of Patients (\%) \\
\hline methicillin-sensitive S. aureus & $23(28)$ \\
methicillin-resistant S. aureus & $23(28)$ \\
coagulase-negative Staphylococcus spp & $9(11)$ \\
Streptococcus spp & $4(5)$ \\
Enterococcus & $3(4)$ \\
Pseudomonas & $2(2)$ \\
Escherichia coli & $2(2)$ \\
Candida albicans & $2(2)$ \\
Klebsiella & $1(1)$ \\
Abiotrophia & $1(1)$ \\
unknown & $10(12)$ \\
\hline
\end{tabular}

86.48 weeks. Four patients were lost to follow-up. At the time of latest follow-up evaluation, there were 39 patients in Group 1 (neurologically intact), 15 patients in Group 2 (radiculopathy), 4 patients in Group 3 (paraplegia or quadriplegia), and 20 patients dead (Group 4). Comparing their neurological status at last follow-up to neurological status at admission, the majority of patients (53\%) reported being neurologically "slightly better" or "unchanged," $15 \%$ reported being "significantly better," and $32 \%$ of patients reported being worse or had died. Of patients who died, the cause of death was sepsis in 14 (56\%), complications from end-stage renal disease in $2(8 \%)$, pulmonary embolism in $2(8 \%)$, and myocardial infarction in $2(8 \%)$.

\section{Operative Versus Nonoperative Management}

At baseline, there was no statistically significant difference between the operative and nonoperative cohorts (Table 5). Of the 82 patients, 30 (37\%) were managed operatively. Overall, 12 patients $(15 \%)$ had a good outcome (7 [23.3\%] treated operatively vs 5 [10\%] treated nonoperatively, $\mathrm{p}=0.03)$. Clinical status in 41 patients $(50 \%)$ remained unchanged (10 [33.3\%] treated operatively vs 31 [59.6\%] treated nonoperatively, $\mathrm{p}=0.01$ ), while that in 3 patients $(4 \%)$ worsened (2 [7\%] treated operatively vs 1 [2\%] treated nonoperatively, $\mathrm{p}=0.53$ ]. Overall, 20 patients $(24.4 \%)$ died (9 [30\%] treated operatively vs 11 [21\%] treated nonoperatively, $\mathrm{p}=0.43]$. At initial presentation, $12(15 \%)$ patients were noted to be paraplegic or quadriplegic, of whom 7 were managed operatively and 5 nonoperatively. Of the surgically treated patients, 3 improved neurologically, 2 remained unchanged, and 2 worsened. Of the nonoperatively managed patients, 2 improved neurologically and 3 worsened neurologically.

\section{Dorsal Versus Ventral Abscess Location}

Of the 82 SEAs, 57 (70\%) were ventrally located and $25(30 \%)$ dorsally located. At baseline, there were no statistically significant differences between the two cohorts (Table 6). At initial presentation, $28 \%$ of patients with dorsally located SEAs were classified into Group 3 (paraplegia or quadriplegia) compared with $9 \%$ of patients with ventrally located SEAs $(p=0.06)$. Of the $70 \%$ with
TABLE 5: Baseline characteristics and clinical outcomes of 82 patients managed operatively and nonoperatively

\begin{tabular}{lccc}
\hline & \multicolumn{2}{c}{ No. of Patients (\%) } & \\
\cline { 2 - 3 } Characteristic & Operative & Nonoperative & p Value \\
\hline baseline features & & & \\
no. of patients & 30 & 52 & \\
mean age* & $63 \pm 8.83$ & $65 \pm 8.46$ & 0.42 \\
male & $19(63)$ & $27(52)$ & 0.31 \\
pain & $24(80)$ & $38(73)$ & 0.76 \\
muscle weakness & $20(67)$ & $27(52)$ & 0.30 \\
bladder dysfunction & $3(10)$ & $2(4)$ & 0.06 \\
bowel dysfunction & $1(3.33)$ & $0(0.0)$ & 0.32 \\
encephalopathy & $0(0.0)$ & $4(7.69)$ & 0.04 \\
fever & $14(47)$ & $29(56)$ & 0.31 \\
clinical outcomes based & & & \\
on treatment & & & \\
$\quad$ better & $7(23)$ & $5(10)$ & 0.03 \\
$\quad$ unchanged & $10(33)$ & $31(60)$ & 0.01 \\
$\quad$ worse/death & $11(37)$ & $14(27)$ & 0.43 \\
\hline
\end{tabular}

* Data are presented as the mean \pm SD.

ventrally located SEAs, $37 \%$ underwent surgical decompression compared with $36 \%$ of those with purely dorsal SEAs $(\mathrm{p}=0.95)$. Of note, all operatively managed patients with purely dorsal SEAs underwent simple laminectomy without fusion. In the cohort of patients with ventral SEA, $9(17 \%)$ improved, $29(56 \%)$ remained unchanged, and 17 $(33 \%)$ worsened or died. In the cohort with dorsal SEAs, $3(13 \%)$ improved, $12(48 \%)$ remained unchanged, and 8 $(32 \%)$ worsened or died.

TABLE 6: Baseline characteristics and clinical outcomes of 82 patients with ventral compared with dorsal SEAs

\begin{tabular}{lccc}
\hline & \multicolumn{2}{c}{ No. of Patients (\%) } & \\
\cline { 2 - 3 } \multicolumn{1}{c}{ Characteristic } & Ventral SEA & Dorsal SEA & p Value \\
\hline baseline features & 57 & 25 & \\
no. of patients & $63 \pm 10$ & $66 \pm 9$ & 0.37 \\
age* & $30(53)$ & $16(64)$ & 0.34 \\
male & $41(72)$ & $20(80)$ & 0.53 \\
pain & $29(51)$ & $17(68)$ & 0.19 \\
muscle weakness & $5(10)$ & $1(4)$ & 0.02 \\
bladder dysfunction & $0(0.0)$ & $1(4.0)$ & 0.48 \\
bowel dysfunction & $2(4.0)$ & $2(8.0)$ & 0.46 \\
encephalopathy & $31(54)$ & $12(48)$ & 0.60 \\
fever & & & \\
clinical outcomes based & & & \\
on treatment & $9(17)$ & $3(12)$ & 0.69 \\
$\quad$ better & $29(56)$ & $12(48)$ & 0.94 \\
$\quad$ unchanged & $17(33)$ & $8(32)$ & 0.67 \\
\hline worse/ death &
\end{tabular}

* Data are presented as the mean \pm SD. 


\section{Variables Correlating With Poor Outcomes}

In a univariate analysis, increasing patient age, male sex, baseline neurological deficit, changes in sensory perception, back and leg pain, abscess location, bowel/ bladder dysfunction, and method of treatment were all associated with poor treatment outcomes. Alternatively, patient ethnicity, anatomical location of abscess (cervical, thoracic, lumbar), encephalopathy, fever, white blood cell count at presentation, and offending infectious agent were not associated with poor treatment outcomes.

\section{Correlation Between Pretreatment Variables and Poor Treatment Outcomes}

When included in a multivariate logistic regression model, an increasing baseline level of pain trended toward significance $(\mathrm{p}<0.08)$ and was associated with a poor outcome; the presence of paraplegia or quadriplegia on initial presentation and a dorsally located SEA were independently associated with poor treatment outcomes. However, increasing patient age, male sex, and degree of sensory disturbance were not associated with poor treatment outcomes (Table 7).

\section{Discussion}

In older patients with multiple comorbidities, the diagnosis of an SEA presents multiple challenges; of primary concern is the impending neurological deterioration due to the poor baseline reserve. In the present analysis of patients 50 years of age and older who were treated for spontaneous SEAs, there were no statistically significant differences when comparing combined surgical decompression/intravenous antimicrobial treatment and intravenous antimicrobial therapy alone. Additionally, our studies suggest that increased baseline neurological morbidity and dorsally located abscesses were independently associated with a poor postoperative prognosis compared with other independent variables.

It has been almost 350 years since SEAs were first described by the Italian anatomist Morgagni and since then there has continued to be significant debate on their management in contemporary literature..$^{7,8,17,18,20}$ Although SEAs are a rare pathology, there is an increasing incidence

TABLE 7: Independent preoperative predictors of poor treatment outcomes*

\begin{tabular}{lcc}
\hline \multicolumn{1}{c}{ Variable } & Coefficient & p Value \\
\hline patient age & 0.41 & 0.65 \\
sex & 0.64 & 0.26 \\
pain & 1.65 & 0.08 \\
paresthesia & 0.33 & 0.27 \\
paraplegia/quadriplegia & 4.87 & 0.02 \\
dorsal abscess location & 5.01 & 0.03 \\
\hline
\end{tabular}

* Increasing baseline level of pain, presence of paraplegia or quadriplegia on initial presentation, dorsally located SEA, or nonoperative management of SEA were independently associated with poor treatment outcomes. in older patients due to a high number of comorbidities and risk factors in this subpopulation. ${ }^{15}$ In prior studies, diabetes mellitus, a condition with the highest incidence in the older population, ${ }^{3,14}$ was the primary predisposing comorbidity represented. ${ }^{11}$ Additional pathologies such as chronic obstructive pulmonary disease and hypertension, which disproportionately affect older patients, are also postulated to affect outcomes in the management of SEAs. ${ }^{15}$ To the best of our knowledge, this study represents the first analysis of comparative outcomes in this particular patient subset.

Karikari and colleagues in 2009 first suggested the incorporation of the anatomy of the abscesses in the formulation of a treatment plan. ${ }^{11}$ For treatment that involves surgical decompression, it would follow, especially in an older population, that the differences in outcomes between ventrally and dorsally located abscesses would be a function of postoperative spinal stability. Specifically, Karikari and colleagues assumed that the preservation of the posterior longitudinal ligament via the ventral approach would reduce postoperative morbidity in this subgroup. As expected, their regression model demonstrated comparatively poorer outcomes in the cases of dorsally located abscesses relative to those of ventrally located lesions. Whether this difference in outcomes can be impacted by specific comorbidities in the older population remains to be seen and warrants further study. In Karikari and colleagues' analysis of 104 patients, there was no added benefit of early surgical management compared with conservative treatment, which was analogous to our findings in an older patient cohort.

In a recent retrospective series comparing the two treatment strategies, Connor and colleagues analyzed data obtained in 77 patients undergoing either conservative management or early surgical decompression. ${ }^{6}$ While baseline neurological dysfunction was directly correlated with assessed outcomes in their study, the authors demonstrated utility of early combined surgical intervention and antimicrobial therapy as an optimal treatment compared with a nonoperative approach. Not surprisingly, patients who fared better at discharge were on average younger than those who did not fare as well, and the difference was statistically significant $(\mathrm{p}=0.04)$. In our cohort, a minority of patients $(14.6 \%)$ reported improvement after therapy with a slight bias toward the operative group ( $\mathrm{p}$ $=0.03)$. The vast majority of patients $(>85 \%)$, however, were either unchanged or worse, with no statistically significant difference between treatment cohorts.

Studies have consistently demonstrated that relative to postoperative outcomes after spinal instrumentation, the presence of a baseline neurological deficit is a strong predictor of patient satisfaction and time to functional independence..$^{5,9,12}$ This is even more significant in the older population in which decreased bone quality combined with multiple comorbidities adversely impacts postoperative outcomes, even in the short term. ${ }^{2,13}$ Similarly, we found that the presence of paraplegia or quadriplegia had an impact almost 3 times that of pain in predicting postoperative outcomes (Table 7).

The limitations inherent in our study have implications for its interpretation. First, this study was limited 


\section{Management of spinal epidural abscesses in older patients}

by its retrospective design. Second, the lack of validated patient-reported outcomes precludes us from making any firm conclusions about the long-term clinical effectiveness of either approach. Additionally, the construction of our study hinges on institutional experience where, at around the age of 50, patients present with multiple comorbidities that differentially affect posttreatment outcomes. Similarly age-matched cohorts may show disparate postoperative functional outcomes due to different perspectives on pain and functional independence or improved comorbidity profiles. Nonetheless, our findings suggest that in older patients early surgical intervention was not associated with superior postoperative clinical outcomes: this finding resonates with earlier evidence of the similarity of conservative medical management in the general patient population. This is an important finding in this patient cohort because, for the operating surgeon, the choice between surgical intervention and conservative management is often counterbalanced by the delicate nature of a patient's comorbidities and compounded by the relative lack of literature to guide these choices. Given the rapid increase in this subpopulation of patients, the decision to surgically intervene or treat conservatively will continue to pose a challenge to neurosurgeons.

\section{Conclusions}

Our study suggests that in patients 50 years of age and older early surgical decompression combined with intravenous antimicrobial therapy was not associated with superior clinical outcomes when compared with intravenous antimicrobial therapy alone.

\section{Disclosure}

Dr. Issacs reports being a consultant for and a stockholder in $\mathrm{NuVasive.} \mathrm{He} \mathrm{also} \mathrm{reports} \mathrm{having} \mathrm{received} \mathrm{clinical} \mathrm{or} \mathrm{research} \mathrm{sup-}$ port from NuVasive.

Author contributions to the study and manuscript preparation include the following. Conception and design: Adogwa, Karikari. Acquisition of data: Adogwa, Karikari, Fatemi, Perez. Analysis and interpretation of data: Adogwa, Karikari, Carr, Krucoff, Ajay, Perez. Drafting the article: Adogwa, Karikari, Carr, Krucoff, Ajay, Cheng. Critically revising the article: all authors. Reviewed submitted version of manuscript: all authors. Approved the final version of the manuscript on behalf of all authors: Adogwa. Statistical analysis: Adogwa, Isaacs. Administrative/technical/material support: Cheng, Bagley, Isaacs. Study supervision: Cheng, Bagley, Isaacs.

\section{References}

1. Baker AS, Ojemann RG, Swartz MN, Richardson EP Jr: Spinal epidural abscess. N Engl J Med 293:463-468, 1975

2. Bernick S, Cailliet R: Vertebral end-plate changes with aging of human vertebrae. Spine (Phila Pa 1976) 7:97-102, 1982

3. Centers for Disease Control and Prevention: National Diabetes Fact Sheet, 2011. Atlanta: US Department of Health and Human Services, 2011 (http://www.cdc.gov/diabetes/pubs/pdf/ ndfs_2011.pdf) [Accessed November 19, 2013]

4. Chen SH, Chang WN, Lu CH, Chuang YC, Lui CC, Chen SF, et al: The clinical characteristics, therapeutic outcome, and prognostic factors of non-tuberculous bacterial spinal epidural abscess in adults: a hospital-based study. Acta Neurol Taiwan 20:107-113, 2011

5. Cobo Soriano J, Sendino Revuelta M, Fabregate Fuente M, Cimarra Díaz I, Martínez Ureña P, Deglané Meneses R: Predictors of outcome after decompressive lumbar surgery and instrumented posterolateral fusion. Eur Spine J 19:1841-1848, 2010

6. Connor DE Jr, Chittiboina P, Caldito G, Nanda A: Comparison of operative and nonoperative management of spinal epidural abscess: a retrospective review of clinical and laboratory predictors of neurological outcome. Clinical article. J Neurosurg Spine 19:119-127, 2013

7. Curry WT Jr, Hoh BL, Amin-Hanjani S, Eskandar EN: Spinal epidural abscess: clinical presentation, management, and outcome. Surg Neurol 63:364-371, 2005

8. Darouiche RO: Spinal epidural abscess. N Engl J Med 355: 2012-2020,2006

9. Fokter SK, Yerby SA: Patient-based outcomes for the operative treatment of degenerative lumbar spinal stenosis. Eur Spine J 15:1661-1669, 2006

10. Hlavin ML, Kaminski HJ, Ross JS, Ganz E: Spinal epidural abscess: a ten-year perspective. Neurosurgery 27:177-184, 1990

11. Karikari IO, Powers CJ, Reynolds RM, Mehta AI, Isaacs RE: Management of a spontaneous spinal epidural abscess: a singlecenter 10-year experience. Neurosurgery 65:919-924, 2009

12. Katz JN, Stucki G, Lipson SJ, Fossel AH, Grobler LJ, Weinstein $\mathrm{JN}$ : Predictors of surgical outcome in degenerative lumbar spinal stenosis. Spine (Phila Pa 1976) 24:2229-2233, 1999

13. Koutsoumbelis S, Hughes AP, Girardi FP, Cammisa FP Jr, Finerty EA, Nguyen JT, et al: Risk factors for postoperative infection following posterior lumbar instrumented arthrodesis. J Bone Joint Surg Am 93:1627-1633, 2011

14. Pereira CE, Lynch JC: Spinal epidural abscess: an analysis of 24 cases. Surg Neurol 63 (Suppl 1):S26-S29, 2005

15. Pradilla G, Ardila GP, Hsu W, Rigamonti D: Epidural abscesses of the CNS. Lancet Neurol 8:292-300, 2009

16. Reihsaus E, Waldbaur H, Seeling W: Spinal epidural abscess: a meta-analysis of 915 patients. Neurosurg Rev 23:175-205, 2000

17. Rigamonti D, Liem L, Sampath P, Knoller N, Namaguchi Y, Schreibman DL, et al: Spinal epidural abscess: contemporary trends in etiology, evaluation, and management. Surg Neurol 52:189-197, 1999

18. Savage K, Holtom PD, Zalavras CG: Spinal epidural abscess: early clinical outcome in patients treated medically. Clin Orthop Relat Res 439:56-60, 2005

19. Sendi P, Bregenzer T, Zimmerli W: Spinal epidural abscess in clinical practice. QJM 101:1-12, 2008

20. Siddiq F, Chowfin A, Tight R, Sahmoun AE, Smego RA Jr: Medical vs surgical management of spinal epidural abscess. Arch Intern Med 164:2409-2412, 2004

21. Zimmerer SM, Conen A, Müller AA, Sailer M, Taub E, Flückiger U, et al: Spinal epidural abscess: aetiology, predisponent factors and clinical outcomes in a 4-year prospective study. Eur Spine J 20:2228-2234, 2011

Manuscript submitted June 7, 2013.

Accepted November 18, 2013.

Please include this information when citing this paper: published online December 20, 2013; DOI: 10.3171/2013.11.SPINE13527.

Address correspondence to: Owoicho Adogwa, M.D., M.P.H., Division of Neurosurgery, P.O. Box 3087, Duke University Medical Center, Durham, NC 27710. email: owoicho.adogwa@gmail.com. 